\title{
Review-Artikel: Wirtschafts- und sozialpolitische Weichenstellungen in der Bundesrepublik Deutschland $^{* *}$
}

Design und Erfolg des „,deutschen Modells“ wurden maßgeblich von zwei wirtschafts- und sozialpolitischen Weichenstellungen nach 1945 bestimmt: der Einführung der Sozialen Marktwirtschaft und der Mitbestimmung der Arbeitnehmer in Betrieb und Unternehmen.

Über beide Phänomene wurde eine Vielzahl von Publikationen veröffentlicht. Wenn hier nun zwei weitere Bücher zu den beiden Gegenstandsbereichen vorgestellt werden, dann aus den nachvollziehbaren Gründen, weil die Publikation zur Sozialen Marktwirtschaft deren Entstehungsgeschichte neu erzählt und weil das Werk zur Arbeitnehmermitbestimmung die Entstehungsgeschichte des Gesetzes von 1976 behandelt, zu der bisher keine fundierte historische Monographie vorliegt, im Gegensatz zu der reichhaltigen Literatur zu den Gesetzen zur Montanmitbestimmung und zur Betriebsverfassung.

Uwe Fuhrmann: Die Entstehung der „Sozialen Marktwirtschaft“ 1948/49. Eine Dispositivanalyse.

UKV Verlagsgesellschaft, Konstanz und München 2017, 359 Seiten, kart., 39,00 Euro.

\section{Christian Testorf: Ein heißes Eisen. Zur Entstehung des Gesetzes über die Mitbe- stimmung der Arbeitnehmer von 1976.}

Reihe Politik und Gesellschaftsgeschichte, Bd. 103. Verlag J.H.W Dietz Nachf., Bonn 2017, 464 Seiten, br., 42,00 Euro.

$* * *$

Mit einem von der Hans-Böckler-Stiftung geförderten Dissertationsprojekt hat der Berliner Historiker Uwe Fuhrmann die Entstehungsgeschichte der Sozialen Marktwirtschaft neu geschrieben. Seine Neuerzählung dürfte eine zentrale Legende aus der Gründungszeit der Bundesrepublik um den „Vater“ der Sozialen Marktwirtschaft, Ludwig Erhard, zurechtrücken. Nach eingängigem Quellenstudium und gründlicher Analyse kommt die mit einem

* Prof. Dr. Walther Müller-Jentsch ist em. Professor für Soziologie an der Ruhr-Universität Bochum. E-Mail: w.mueller-jentsch@rub.de

** Artikel eingegangen: 24.12.2017, revidierte Fassung nach doppelt-blindem Begutachtungsverfahren akzeptiert: 2.4 .18 
ambitionierten wissenschaftlichen Instrumentarium durchgeführte Untersuchung zu überraschenden Ergebnissen. Zu diesen gehört, dass ein Sozialdemokrat, Leonhard Miksch, der dem ordoliberalen Kreis um Walter Eucken angehörte, maßgeblich am Konzeptionswechsel von der „freien“ zur ,sozialen“ Marktwirtschaft beteiligt war.

Aber schauen wir etwas genauer auf die Diskurse und Ereignisse der späten 1940er Jahre.

Alfred Müller-Armack, der als Wortschöpfer der „,sozialen Marktwirtschaft“ gilt (Abelshauser, 1991, S. 21), hatte im Januar 1947 die Schrift „Wirtschaftslenkung und Marktwirtschaft" veröffentlicht, in der er sich über das Scheitern der liberalen Marktwirtschaft und sein Konzept der sozialen Marktwirtschaft auslässt, das eine wirksame Sozialpolitik (inklusive Mindestlohn!) einschloss. Fuhrmann schreibt ihm in diesen Jahren eine marginale Rolle zu, da er erst 1952 in Erhards Wirtschaftsministerium zum Leiter der Grundsatzabteilung berufen wurde. Dies scheint mir nicht gerechtfertigt, zählte er doch bereits Anfang 1948 zu den Gründungsmitgliedern des Wissenschaftlichen Beirats ${ }^{1}$ des Frankfurter Wirtschaftsrats (einer Art vorläufiger Regierung für die Westzonen). Auch hatte er bereits 1948 einen anspruchsvollen programmatischen Entwurf - „Vorschläge zur Verwirklichung der Sozialen Marktwirtschaft“ (Müller-Armack, 1948) - veröffentlicht.

Der im März 1948 zum Direktor für das Ressort Wirtschaft des Frankfurter Verwaltungsrats $^{2}$ gewählte Ludwig Erhard verfolgte den Plan, mit der bevorstehenden Währungsreform und anschließender Freigabe der Konsumgütermärkte eine „freie Marktwirtschaft“ zu etablieren. Fuhrmann nennt den Plan nach Foucault ein „strategisches Dispositiv“ (mehr dazu später).

Leonhard Miksch, der seit Anfang 1948 als Leiter des Referats für „Preiswirtschaftliche Grundsatzfragen und Betriebswirtschaft“ zum engen Mitarbeiter Erhards wurde, verwendete den Begriff soziale Marktwirtschaft lange vor Erhard, so Ende 1947 in der Fachzeitschrift „Der Wirtschaftsspiegel“ und im Januar 1948 in einer internen Denkschrift, ,in der er die sprachliche Wende von der ,freien“ zur ,sozialen“ Marktwirtschaft vollzog“" (S. 145). Dass er dies in Kenntnis der Schrift von Müller-Armack tat, vermutet Fuhrmann, doch ist das mehr als eine Vermutung (siehe oben). Eine öffentliche Verwendung des Begriffs ist laut Fuhrmann jedoch nicht vor Juni 1948 belegt (S. 261).

Grundlegend für die neue Wirtschaftsordnung, um deren genaue Bezeichnung (,freie“ oder „soziale Marktwirtschaft“"?) im Verlaufe des Jahres 1948 noch gestritten wurde, war die „Doppelreform“ von Währungsreform vom 20. Juni 1948 und von dem zur gleichen Zeit erlassenen Leitsätzegesetz, ${ }^{3}$ das dem Direktor für Wirtschaft, also Erhard, ,weitreichende Vollmachten“ gab, ,große Gruppen von Waren nach eigenem Ermessen von der Preisbindung zu befreien“ (S. 144).

1 Der Beirat hatte 17 Gründungsmitglieder, darunter die Professoren Franz Böhm, Walter Eucken, Alfred Müller-Armack, Erik Nölting, Oswald v. Nell-Breuning, Erich Preiser, Karl Schiller, Otto Veit und Gerhard Weisser.

2 Als ausführendes Organ hatte der Wirtschaftsrat einen Verwaltungsrat installiert mit den Ressorts Wirtschaft - Ernährung, Landwirtschaft und Forsten - Finanzen - Verkehr - Post- und Fernmeldewesen - Arbeit, die alle von CDU/CSU-Vertretern geleitet wurden. Nur Erhard war als Ressortleiter für Wirtschaft der einzige Parteilose (Lehmann, 1981, S. 23).

3 Die genaue Bezeichnung des Gesetzes lautete: „Leitsätze für die Bewirtschaftung und Preispolitik nach der Währungsreform“. 
Wie Fuhrmann aufzeigt, stieß Erhards Vorgehen auf erheblichen Widerstand. Die Preise explodierten. ${ }^{4}$ „Runter mit den Preisen und fort mit Erhard, hieß es“ (Hentschel, 1998a, S. 89). In der CDU waren vornehmlich die Sozialausschüsse mit dem rein marktwirtschaftlichen Kurs unzufrieden; sie verwiesen auf den Widerspruch zwischen einer „freien Marktwirtschaft" und dem Ahlener Programm. ${ }^{5}$ Von der SPD wurden im Frankfurter Wirtschaftsrat zwei (erfolglose) Misstrauensanträge eingebracht (17. August und 10. November 1948). Zahlreiche Protest- und Käuferstreiks und nicht zuletzt ein eintägiger Generalstreik (12. November 1948) richteten sich gegen die inflationären Folgen der Preisfreigabe.

Tatsächlich sah sich Erhard zu einigen nicht-marktwirtschaftlichen Korrekturen gezwungen. Dazu zählten das Gesetz gegen Preistreiberei und der Preisspiegel. Das Gesetz sollte „,besonders krasse Fälle von Preiswucher, bei Warenzurückhaltung und Warenvernichtung“ (S. 234) sanktionieren; die periodisch herausgegebenen Listen des Preisspiegels sollte Verbrauchern Orientierung beim Einkauf liefern. Beide Maßnahmen waren von begrenzter Wirkung; aber ihre symbolische Bedeutung bestand in dem Eingeständnis, dass es nötig war, die Preise politisch zu beeinflussen, was wiederum von den Kritikern ,als eine Art Offenbarungseid des Strategischen Dispositivs ,freie Marktwirtschaft” bewertet“" (S. 235) wurde.

Des Weiteren zählte zu den Korrekturen die Waren-Lenkung von Rohstoffen und Konsumgütern. Unter dem Titel „Jedermann-Programm“ wies der Staat jenen Firmen Rohstoffe zu, die ,Jedermannwaren“ (wie z.B. Schuhe) am billigsten und schnellsten auf den Markt zu bringen vermochten. Das Konzept für dieses Programm hatte Miksch erarbeitet.

Auf der Diskursebene bemächtigte sich eine vielfältige Opposition von christlicher Soziallehre, Vertretern der SPD und Gewerkschaften des Begriffs der sozialen Marktwirtschaft. Fuhrmann spricht von einer verdeckten, nicht gemeinsam auftretenden Allianz von „,institutionalisierter Arbeiterbewegung und christlicher Soziallehre“ (S. 298), der es in der zweiten Hälfte des Jahres 1948 gelungen war, die „soziale Marktwirtschaft“ erfolgreich gegen den Kurs Erhards einzufordern.

Somit kommt Fuhrmann zu dem nachvollziehbaren Schluss, dass SPD und DGB der „Sozialen Marktwirtschaft“ den Weg bereiteten (S. 281), indem sie wirkungsmächtig Einfluss auf Diskurse über die Wirtschaftsordnung und auf die reale Marktpolitik nahmen. Schließlich war es aber Erhard, der angesichts der neuen Kräfteverhältnisse erstmals im Februar 1949 seine Politik einschließlich ihrer Korrekturen mit der opportunistischen Übernahme des Begriffs der Sozialen Marktwirtschaft ausflaggte (S. 300), was ihm „die Möglichkeit gab, als Protagonist einer Heldenerzählung in die Geschichte einzugehen“ (S. 121). Weitsichtig hat Alfred Weber schon früh in einem Aufsatz für die „Gewerkschaftlichen Monatshefte“ beklagt: „Ich bedaure es, dass sich der deutsche Sozialismus das Wort

4 Der Index der Lebenshaltungskosten stieg in den ersten vier Monaten nach der Währungsreform um 14 Prozent, vermerkt der Erhard-Biograf Volker Hentschel (1998a, S. 87).

5 Das „Ahlener Programm“ ist ein von der nordrhein-westfälischen CDU am 3. Februar 1947 beschlossenes Wirtschafts- und Sozialprogramm, dessen Kernaussage lautete: „Das kapitalistische Wirtschaftssystem ist den staatlichen und sozialen Lebensinteressen des deutschen Volkes nicht gerecht geworden. " Die als christlicher Sozialismus verstandenen Forderungen enthielten die teilweise Vergesellschaftlichung der Großindustrie und weitgehende Mitbestimmungsrechte für die Arbeitnehmer, bei entschiedener Ablehnung eines Staatssozialismus. Mit der Verabschiedung der „Düsseldorfer Leitsätze“ vom 15. Juli 1949, dem CDU-Programm für die erste Bundestagswahl, verabschiedete sich die CDU vom christlichen Sozialismus. 
von der ,sozialen Marktwirtschaft" durch fremde Geschicklichkeit hat wegnehmen lassen“ (Weber, 1950, S. 394).

Warum Sozialdemokratie und Gewerkschaften Erhard die Deutungshoheit über Begriff und Konzeption einer Wirtschaftsordnung überließen, an deren Entstehung sie maßgeblich mitgewirkt hatten, darüber lässt sich Fuhrmann nicht weiter aus. Zu vermuten ist, dass SPD und mehr noch der DGB der Konzeption der „Wirtschaftsdemokratie“ verpflichtet waren, wie es auch Christian Testorf in seiner Studie über das Mitbestimmungsgesetz für die Gewerkschaftsfunktionäre in der Frühphase der Bundesrepublik konstatiert (dort S. 45). Dieses programmatische Erbe der Weimarer Republik gab die SPD mit dem Godesberger Programm (1956) auf; der DGB erkannte erst mit dem Dresdner Programm von 1996 die soziale Marktwirtschaft an. „Die sozial regulierte Marktwirtschaft bedeutet gegenüber einem ungebändigten Kapitalismus einen großen historischen Fortschritt. Die soziale Marktwirtschaft hat einen hohen materiellen Wohlstand bewirkt", heißt es darin.

Begreifen wir, wie es nicht zuletzt ausländische Beobachter tun, die Soziale Marktwirtschaft als die real existierende Wirtschaftsordnung der Bundesrepublik Deutschland, dann kann mit Fug und Recht behauptet werden, dass sie von Gewerkschaften und Sozialdemokratie aktiv mitgeprägt wurde; dies gilt - wie nun die Studie von Fuhrmann dokumentiert bereits für ihre Entstehungsphase.

In ihr spielte der sozialdemokratische Ordoliberale Leonhard Miksch offensichtlich eine bisher unterschätzte Rolle (s. dazu auch Goldschmidt \& Berndt, 2000 und 2005). Er war Erhards enger Mitarbeiter und Ideengeber, gewissermaßen ein Vorläufer Müller-Armacks, der diese Rolle später, ab 1952 ausübte. In seinem wissenschaftlichen Schrifttum argumentierte Miksch gegen den Laissez-faire-Liberalismus und dessen Vorstellung, der Wettbewerb würde sich als „natürliche Ordnung“ herstellen, wenn der Staat sich aus der Wirtschaft heraushalte. Vielmehr sprach er von einer „gesteuerten Wettbewerbsordnung“, vom Wettbewerb als einer „staatlichen Veranstaltung“. Mickschs Entwurf über die „Grundsätze der Wirtschaftspolitik für die der Währungsunion folgende Übergangsperiode“ deckte sich inhaltlich ,in erstaunlichem Maße mit dem [...] beschlossenen tatsächlichen [Leitsätze-] Gesetz" (S. 148). Dies veranlasste den Historiker Gerold Ambrosius zu der Bemerkung: „Es entbehrt nicht der Ironie, daß ausgerechnet der Gesetzentwurf, der den weiteren Aufbau der Westzonen und der Bundesrepublik unter christlich-demokratischer Führung als auch die Wirtschaftsprogrammatik der Union entscheidend beeinflussen sollte, von einem Sozialdemokraten entworfen wurde“ (Ambrosius, 1977, S. 173). Die Ersetzung des Begriffs „freie Marktwirtschaft“ durch „,soziale Marktwirtschaft“ ist, wie bereits erwähnt, auf Miksch zurückzuführen; dessen sozialen Gehalt hat dann sein späterer Nachfolger im Bundeswirtschaftsministerium, Müller-Armack, programmatisch aufgeladen. Miksch wie MüllerArmack dachten weiter als Erhard, den sein Biograf, Volker Hentschel, als einen „begnadeten Populisten“ (Hentschel, 1998b, S. 22) charakterisiert. Bei den Gewerkschaften stieß Miksch indes auf keine Gegenliebe. In einem Schreiben des Gewerkschaftsrates der Vereinigten Zonen an seine Mitglieder wurde er 1949 als „Schleppenträger Erhardscher Wirtschaftspolitik“ bezeichnet (Ambrosius, 1977, S. 266, Anm. 155).

Fuhrmanns Neuerzählung der Entstehung der Sozialen Marktwirtschaft erfolgt in einem von Foucault übernommen theoretischen Bezugsrahmen, der kurz gefasst wie folgt aussieht: 
Zentrale Rolle kommt der Kategorie des Dispositivs zu. Darunter versteht Foucault in der Interpretation von Fuhrmann ein Netz von verschiedenen (diskursiven und nichtdiskursiven) Elementen mit einer strategischen Funktion. Die strategische Zielsetzung ist darauf gerichtet, einen Notstand im Sinne bestimmter Interessen oder Überzeugungen aufzulösen. Als „strategisches Dispositiv“ gilt hier das interessengeleitete Vorhaben der Einführung der „freien Marktwirtschaft“, das durch mannigfache - diskursive und nichtdiskursive - Widerstände als Ergebnis der Geschichte zum „modifizierten Dispositiv“ der „Sozialen Marktwirtschaft“ umformuliert wird. Fuhrmann meint, mit dem DispositivBegriff besser Diskurs- und Sozialgeschichte miteinander verknüpfen zu können. Einem anderen theoretischen Kontext entnahm Fuhrmann den Begriff des „Leeren Signifikanten“ als „Platzhalter des Allgemeinen“; so wurde der Begriff der "Sozialen Marktwirtschaft" von Erhard erstmals in einer öffentlichen Rede (17. August 1948) für eine Wirtschaftsordnung des größten Allgemeinwohls kommuniziert (S. 264f.), während „freie Marktwirtschaft" für die Zeitgenossen zu sehr als Ersatzbezeichnung für den diskreditierten Kapitalismus lesbar war.

Einen empfehlenden Hinweis möchte ich noch auf eine bereits 2011 erschienene Dissertation geben:

\section{Gero Thalemann: Die Soziale Marktwirtschaft der Bundesrepublik Deutschland - ein realisiertes Konzept? Analyse von Genesis, theoretischem Gehalt und praktischer Verwirklichung.} disserta Verlag, Hamburg 2011, 235 + LXV Seiten, br., 49.50 Euro.

Mit dieser Publikation unternimmt der Autor den Versuch, den Anspruch der Sozialen Marktwirtschaft mit der wirtschafts-und sozialpolitischen Wirklichkeit zu konfrontieren. In einer aufwändigen empirischen Überprüfung der Grundwerte und Ziele anhand einschlägiger Daten und Kennziffern von 1950 bis in die Gegenwart kommt der Autor zu der Schlussfolgerung, ,dass die Soziale Marktwirtschaft in der Bundesrepublik Deutschland als ein realisiertes Konzept betrachtet werden kann“ (S. 235).

Thalemanns und Fuhrmanns Publikationen werfen auf das Thema Soziale Marktwirtschaft neues Licht: Fuhrmann, indem er ihre Genese differenzierter als frühere Autoren ausleuchtet; Thalemann, indem er der realen Wirtschaftsordnung Deutschlands dieses Attribut auch heute noch zu Recht zuerkennt.

$$
* * *
$$

Die Entstehungsgeschichte des Mitbestimmungsgesetzes 1976 ist aus heutiger Sicht kaum weniger verzwickt als die der Sozialen Marktwirtschaft. In den späten 1960er und den 1970er schien der DGB der politischen Realisierung seiner Forderung, die 1951 in der Montanindustrie gesetzlich eingeführte paritätische Unternehmensmitbestimmung auf alle übrigen Wirtschaftssektoren auszudehnen, nahegekommen zu sein. Das Parlament verabschiedete am 18. März 1976 ein Mitbestimmungsgesetz, das auf einem Kompromiss zwischen den Koalitionsparteien SPD und FDP beruhte, aber gleichwohl von einer großen parlamentarischen Mehrheit angenommen worden war. Jedoch kommentierte es der damalige DGB-Vorsitzende, Heinz Oskar Vetter, als „größte Enttäuschung meiner Amtszeit“. Der Grund für Vetters Verärgerung war die Verwässerung des Paritätsgedankens. Denn dem 
Vorsitzenden des Aufsichtsrats wurde in Pattsituationen ein doppeltes Stimmrecht eingeräumt, und zudem wurde den Vertretern der Arbeitnehmer ein leitender Angestellter zugesellt.

Testorfs Monographie holt weit aus. Nach den einleitenden Abschnitten zur Fragestellung, Methodik und zum Forschungsüberblick folgt ein gesondertes Kapitel mit einem historischen Abriss der Mitbestimmungsgeschichte vom Vormärz bis zum Montanmitbestimmungs- und Betriebsverfassungsgesetz Anfang der 1950er Jahre. Mit diesen beiden Gesetzen, vornehmlich mit dem Mitbestimmungsgesetz für die ehemals autokratisch und paternalistisch geführten Unternehmen der Montanindustrie, wurde in Deutschland der entscheidende neue Pfad zur betrieblichen Sozialpartnerschaft gelegt. Dem Ruhrgebiet als der damals dominanten westdeutschen Industrieregion fiel auch eine sozialpolitische Vorreiterrolle zu (Müller-Jentsch, 1998).

Nach der vom DGB empfundenen Niederlage durch die Verabschiedung des Betriebsverfassungsgesetzes 1952, weil es für die Aufsichtsräte großer Kapitalgesellschaften nur eine Drittelbeteilung vorsah, nahmen erst Mitte der 1960er Jahre die innergewerkschaftlichen Debatten über die Ausweitung der Unternehmensmitbestimmung wieder Fahrt auf. Sie schlugen sich nieder in der Aktualisierung des Aktionsprogramms vom März 1965 und in der im Mai 1966 vorgelegten Denkschrift „Mitbestimmung - eine Forderung unserer Zeit“. In ihr hoben die Verfasser hervor, dass die deutsche Form der Mitbestimmung ,das Gegenstück zum Klassenkampf französischer und italienischer Gewerkschaften“ (S. 129) darstelle und forderte vom Gesetzgeber ,eine Ausweitung der Mitbestimmung in vergleichbarer Form zur Montanindustrie“ (S. 130).

Die Unternehmer gingen ihrerseits in die Verteidigung des Status quo. Zwei bemerkenswerte Stellungnahmen aus jenen Jahren seien hervorgehoben. Hanns-Martin Schleyer lobte 1967 auf einem Wirtschaftstag der CDU/CSU das Betriebsverfassungsgesetz über den grünen Klee als Kompensation für die Unternehmensmitbestimmung.

„Die empirisch-soziologischen Untersuchungen [...] haben gezeigt, welcher großen Wertschätzung sich der Betriebsrat innerhalb der Belegschaften erfreut und wie beträchtlich sein Anteil an der Aufrechterhaltung des sozialen Friedens in den Betrieben ist. Deshalb bekennen wir uns uneingeschränkt zu diesem Betriebsverfassungsgesetz, das in der gesamten westlichen Welt kein ebenbürtiges Vorbild hat, zu einem Gesetz, das die soziale Partnerschaft und die Menschenwürde des Arbeitnehmers zu seinen Grundprinzipien erhoben hat" (139f.).

Das publizistische Sprachrohr der deutschen Industrie, der „Industriekurier“, polemisierte indessen:

„Die Demokratisierung der Wirtschaft ist so unsinnig wie eine Demokratisierung der Schulen, der Kasernen und der Zuchthäuser (Industriekurier vom 7. Oktober 1967; hier zitiert nach Testorf, S. 140).

Mit „Erste Anläufe“ überscheibt Testorf das Kapitel, das sich mit der Zeit der Großen Koalition, 1966 bis 1969, befasst. Ausführlich schildert er die Debatten in den Parteien und Gewerkschaften über neue Initiativen zur Mitbestimmung, die Kontroversen zwischen Wirtschaftsrat und Sozialausschüssen in der CDU, zwischen SPB und DGB.

Wichtigstes Ereignis in diesen Jahren war die Einsetzung einer Regierungskommission („Biedenkopf-Kommission“) zur Auswertung der bisherigen Erfahrungen mit der Mitbe- 
stimmung. Die sich im Januar 1968 konstituierte Kommission war ein reines Professorengremium. Neben den neun Professoren (Kurt Biedenkopf, Kurt Ballerstedt, Erich Gutenberg, Harald Jürgensen, Wilhelm Krelle, Ernst-Joachim Mestmäcker, Rudolf Reinhardt, Fritz Voigt, Hans Willgerodt) entsandten Wirtschaftsunternehmen, Gewerkschaften und Arbeitgeberverbände sechs ständige Berater. Die Ergebnisse der Kommission wurden im Januar 1970 vorgelegt. Grundsätzlich äußerte sich der Bericht positiv über die Erfahrungen mit der Montanmitbestimmung und empfahl eine Ausweitung der Unternehmensmitbestimmung auf große Kapitalgesellschaften der übrigen Wirtschaft. Die Zahl der Arbeitnehmervertreter sollte über die bestehende Drittelbeteilung hinaus vermehrt werden, allerdings unter Beibehaltung eines geringen Übergewichts der Anteilseigner. Auch Gewerkschaftsvertreter seien erwünscht. Bis auf die streitige Frage der Parität begrüßte der DGB die Empfehlungen. Bemerkenswert war, dass der Bericht einen ersten ordnungspolitischen Versuch enthielt, die Mitbestimmung in das marktwirtschaftliche System zu integrieren (MüllerJentsch, 2011, S. 151). Die Notwendigkeit von Mitbestimmung leitete er ,,aus dem ,besonderen rechtlichen, wirtschaftlichen und sozialen Charakter des Arbeitsverhältnisses‘ sowie aus der ,Zugehörigkeit des Arbeitnehmers zum Unternehmen“ ab“ (S. 281).

Im Oktober 1969 war nach einem Wahlsieg der SPD eine sozialliberale Regierungskoalition zwischen SPD und FDP unter dem Kanzler Willy Brandt gebildet worden. Daraus ergaben sich neue Bedingungen und Chancen für ein Mitbestimmungsgesetz. Mit ihren Freiburger Thesen von 1971 vollzog die FDP eine programmatische Umorientierung; neben den traditionellen liberalen Freiheitsrechten betonten die Thesen nun auch wirtschaftliche und soziale Teilhaberechte, eine „Demokratisierung der Gesellschaft“ und „Reform des Kapitalismus“. Gleichwohl lagen die Vorstellungen von SPD und FDP in Bezug auf die Unternehmensmitbestimmung noch auseinander. Die FDP bestand auf der Einbeziehung der leitenden Angestellten. Die SPD sah sich in der Frage der Parität gegenüber dem DGB in der Pflicht. Zunächst konnten beide Parteien sich auf eine Novellierung des Betriebsverfassungsgesetzes einigen, die mit substantiell erweiterten Rechten für die Betriebsräte 1972 in Kraft trat und die Zustimmung des DGB fand.

Das harte Ringen der Akteure um einen Kompromiss zur Unternehmensmitbestimmung wird in dem Buch ausführlich (zuweilen langatmig) dargestellt. Wir erfahren, wie die FDP auf der Sonderstellung der leitenden Angestellten, der Wahl von betriebsfremden Gewerkschaftern durch die Belegschaft und auf einem Übergewicht der Kapitalseite beharrte, wie die Beziehungen zwischen SPD und DBG sich trübten, wie ein Entwurf (1974) durch den nächsten (1976) ersetzt wurde, bis man zur Abstimmung kam. Wen immer das im Detail interessiert, der kommt voll auf seine Kosten.

Verwunderlich bleibt, dass letztlich der Kompromiss parteienübergreifend mit nur 22 Gegenstimmen angenommen wurde. Und die kamen, bis auf eine Ausnahme, vom Wirtschaftsflügel der CDU. Der Arbeitsminister Walter Arendt, ehemaliger Vorsitzender der IG Bergbau, lobte das längst fällige Gesetz als eine Grundlage für eine faire Zusammenarbeit zwischen Kapital und Arbeit, machte aber keinen Hehl aus seiner Enttäuschung über die unterparitätische Mitbestimmung, die letztlich der wirtschaftsliberale Flügel der FDP um Graf Lambsdorff durchgesetzt hatte.

Wenn auch der DGB das Gesetz als eine Niederlage begriff, nutzte er die neugeschaffenen Möglichkeiten, die insbesondere die Position der Betriebsräte stärkten, da sie nun in 
Personalunion gleichzeitig einen Sitz Aufsichtsrat einnehmen konnten. Von 29 Arbeitgeber- und Wirtschaftsverbände wurde zusammen mit neun Unternehmen eine Verfassungsbeschwerde gegen das Gesetz eingereicht, mit der Begründung, dass es die Eigentumsgarantie des Grundgesetzes verletze. Das Bundesverfassungsgericht wies die Beschwerde unter anderem mit dem Hinweis auf das „Übergewicht, welches das Gesetz der Anteilseignerseite einräumt“, zurück. Das Gesetz verstoße auch nicht gegen die grundgesetzliche Eigentumsgarantie und überdies sei das Grundgesetz wirtschaftspolitisch neutral; es enthalte keine ,unmittelbare Festlegung und Gewährleistung einer bestimmten Wirtschaftsordnung“. Nach diesen eindeutigen Worten des Bundesverfassungsgerichts lernten die Kontrahenten in den folgenden Jahren, sich mit der anfänglich beiderseitig ungeliebten Unternehmensmitbestimmung zu arrangieren. Heute gilt sie als „,wesentliches Element der Sozialen Marktwirtschaft“" - so das Urteil der Kanzlerin Angela Merkel auf der DGB- Jubiläumsveranstaltung zur 30jährigen Verabschiedung des Gesetzes.

Das Buch von Testorf schließt eine Lücke in der Mitbestimmungsliteratur. Sein Verdienst bliebe ungeschmälert, wenn es das Lektorat des 463 Seiten umfassenden Bandes nicht versäumt hätte, ihm ein Personen- und Sachregister beizufügen. Bei wissenschaftlichen Publikationen dieser Art ist dies ein zu erwartendes Desiderat.

\section{Literatur}

Abelshauser, W. (1991). Die ordnungspolitische Epochenbedeutung der Weltwirtschaftskrise in Deutschland: Ein Beitrag zur Entstehungsgeschichte der Sozialen Marktwirtschaft. In D. Petzina (Hrsg.), Ordnungspolitische Weichenstellungen nach dem Zweiten Weltkrieg. Schriften des Vereins für Socialpolitik, Neue Folge Bd. 203 (S. 11-29). Berlin: Duncker \& Humblot.

Ambrosius, G. (1977). Die Durchsetzung der Sozialen Marktwirtschaft in Westdeutschland 19451949. Stuttgart: Deutsche Verlags-Anstalt.

Goldschmidt, N. \& Berndt, A. (2000). „Wettbewerb als Aufgabe“ - Leonhard Mikschs Beitrag zur Ordnungstheorie und -politik. ORDO: Jahrbuch für die Ordnung von Wirtschaft und Gesellschaft, 51, 33-74.

Goldschmidt, N. \& Berndt, A. (2005). Leonhard Miksch (1901-1950). A Forgotten Member of the Freiburg School. The American Journal of Economics and Sociology, 64 (4), 973-998.

Hentschel, V. (1998a). Ludwig Erhard. Ein Politikerleben. Berlin: Ullstein.

Hentschel, V. (1998b). Ludwig Erhard, die „soziale Marktwirtschaft“ und das Wirtschaftswunder. Historisches Lehrstück oder Mythos? Bonn: Bouvier.

Lehmann, H. G. (1981). Chronik der Bundesrepublik Deutschland 1945/49-1981. München: Beck.

Müller-Armack, A. (1948). Vorschläge zur Verwirklichung der Sozialen Marktwirtschaft. In (1981): Genealogie der Sozialen Marktwirtschaft. Frühschriften und weiterführende Konzepte (2. Aufl.) (S. 91-109). Bern: Haupt.

Müller-Armack, A. (1959). Die Soziale Marktwirtschaft nach einem Jahrzehnt ihrer Erprobung. In E. Tuchtfeldt (Hrsg), (1966). Wirtschaftsordnung und Wirtschaftspolitik. Studien und Konzepte zur Sozialen Marktwirtschaft und zur Europäischen Integration (S. 251-265). Freiburg: Rombach.

Müller-Jentsch, W. (1998). Industrielle Beziehungen in der Region: Das Ruhrgebiet als sozialpolitisches Laboratorium. In H. Hirsch-Kreinsen \& H. Wolf (Hrsg.), Arbeit, Gesellschaft, Kritik. Orientierungen wider den Zeitgeist (S. 151-177). Berlin: Edition Sigma.

Müller-Jentsch, W. (2011). Gewerkschaften und Soziale Marktwirtschaft seit 1945. Stuttgart: Reclam Verlag.

Weber, A. (1950). Sozialistische Marktwirtschaft. In Gewerkschaftliche Monatshefte, 1 (9), S. 394. 
DuEPublico

Duisburg-Essen Publications online
UNIVERSITÄT

$D_{E} U_{S} S_{S} S_{N} U R$ G

offen im Denken

Ub $\begin{aligned} & \text { universitäts } \\ & \text { bibliothek }\end{aligned}$

Dieser Text wird via DuEPublico, dem Dokumenten- und Publikationsserver der Universität Duisburg-Essen, zur Verfügung gestellt. Die hier veröffentlichte Version der E-Publikation kann von einer eventuell ebenfalls veröffentlichten Verlagsversion abweichen.

DOI: $\quad 10.3224 /$ indbez.v25i3.06

URN: urn:nbn:de:hbz:464-20210412-114610-4 4.0) genutzt werden. 